\title{
An SEIRD Epidemic Model for Predicting the Spread of COVID-19 over a Period of One Year: A Case of the United States
}

\author{
Joseph Roger Arhin ${ }^{1, ~ *, ~ F r a n c i s ~ S a m ~}{ }^{1}$, Kenneth Coker $^{2}$, Ernest Owusu Ansah ${ }^{1}$ \\ ${ }^{1}$ School of Mathematical Sciences, University of Electronic Science and Technology of China, Chengdu, China \\ ${ }^{2}$ School of Information and Communication Engineering, University of Electronic Science and Technology of China, Chengdu, China \\ Email address: \\ mailroger2day@gmail.com (J. R. Arhin), fransamang@gmail.com (F. Sam), kennethcoker25@gmail.com (K. Coker), \\ ernest.o.ansah1@gmail.com (E. O. Ansah) \\ ${ }^{*}$ Corresponding author
}

\section{To cite this article:}

Joseph Roger Arhin, Francis Sam, Kenneth Coker, Ernest Owusu Ansah. An SEIRD Epidemic Model for Predicting the Spread of COVID-19 over a Period of One Year: A Case of the United States. American Journal of Mathematical and Computer Modelling.

Vol. 5, No. 3, 2020, pp. 70-76. doi: 10.11648/j.ajmcm.20200503.12

Received: June 26, 2020; Accepted: July 16, 2020; Published: July 28, 2020

\begin{abstract}
COVID-19 is currently a perilous disease that has an incubation period of between 4 and 6 days. The United States Disease Control and Prevention Centers posited that in certain cases, coronaviruses are zoonotic, which means that they have been responsible for moving from animals to humans. The outbreak of the new coronavirus (COVID-19) disease has had an enormous impact globally. The World Health Organization (WHO) has put in place various safety measures that will help alleviate the spread of the epidemic. This paper presents an SEIRD epidemic model with government policy to predict the spread of COVID-19. Through mathematical analysis, the essence of the model is investigated. The basic reproductive number of the envisaged model is computed and decides whether or not the disease is present in the population. Disease-free and symptomatic equilibria are studied for their existence and stability via the Lyapunov function. It is established from our numerical simulations that the introduction of government policy helps to alleviate the spread of the disease, where the basic reproductive number takes part in sustaining their stability. In the prediction of infected and death cases that were very similar to real-life data, it was established that the model was effective.
\end{abstract}

Keywords: COVID-19, SEIRD, Basic Reproduction Number, Disease-free Equilibrium, Routh-Herwitz Criterion,

Global Stability, Lyapunov Function, LaSalle's Invariance Principle

\section{Introduction}

Infectious diseases affect human lives immensely and millions of people have been killed by many infectious diseases. Controlling infectious disease over the years, was a difficult issue [1]. Many of the infectious conditions in nature have been revealed in hosts for a time before hosts become infectious [2]. COVID-19 as an infectious viral disease is first reported in the winter month of December 2019 in a city called Wuhan in Hubei Province of China [3]. 2019-nCov is an epidemic disease caused by a novel virus, which is engendering a global emergency and requires a mathematical model to simulate its spread taking into consideration its notorious distinct characteristics. Thus, it would be expedient to develop a mathematical model which will predict the spread of the virus.

Modelling and simulation are significant decision tools that is used in controlling human and animal diseases [4-7]. Though, since each disease shows its peculiar biological features, various models have been adopted to each distinct instance in order to tackle actual situations $[8,9]$. In the paper of Roosa et. al [10], the authors presented three phenomenological models which confirmed with outbreaks of other forms of infectious diseases other than COVID-19. Several works $[11,12]$, proposed the SEIR models with some variations with stochastic modules. 


\section{The SEIRD Model}

\subsection{Description of the SEIRD Model}

The proposed SEIRD model is a compartmental model used in modelling epidemics like the deadly disease, nCOV-19. The susceptible group over time moves into the exposed (pre-infection) compartment with infection rate, $\beta$. Individuals in the exposed group with a rate at which the infected persons become infectious $\varepsilon$ move into the infectious compartment. The infectious persons may or may not recover at rates $r$ and $d$ respectively. Individuals who may recover after treatment joins the susceptible compartment since there is no vaccine for the disease.

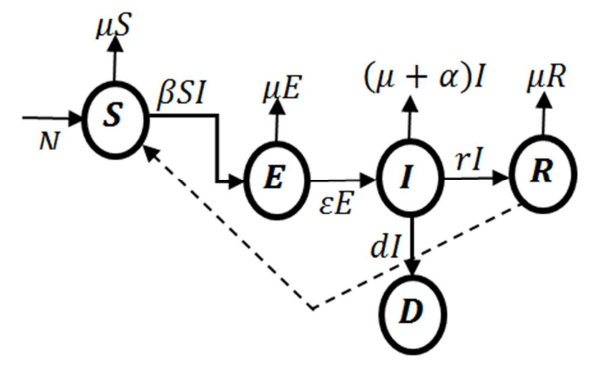

Figure 1. The schematic illustration of the SEIRD model.

Assumption of the SEIRD model includes the following: short, i.e. the population is constant and there are deaths caused by the disease and;

random mixing, in other words, we assume that people behave like air particles and can be expected to contact people far away.

\subsection{Creating the SEIRD Model}

Creating the equation that labels the susceptible group becomes infected at rate $\beta$. Thus, the change of population of the susceptible group is equal to the negative product of the susceptible, $S(t)$ and infectious, $I(t)$ with time, $t$.

$$
\frac{d S}{d t}=-\beta S(t) I(t)
$$

This is incomplete because there is a possibility that a new child will be born or a foreigner travelling into the country at rate a $N$ and there is $\mu$ as natural death in the compartment. The above equation now becomes

$$
\frac{d S}{d t}=N-\beta S(t) I(t)-\mu S(t)
$$

This completes the equation that labels the transformation in the population of the susceptible group with time.

The equation that defines the exposed group begins by adding what was just removed from the susceptible group, $\beta S(t) I(t)$

$$
\frac{d E}{d t}=\beta S(t) I(t)-\mu E(t)-\varepsilon E(t)
$$

where $\varepsilon$ is the extent whereby an affected person becomes infectious per unit time and $\mu$ is the natural death rate.

Individuals that are exposed then join the infectious compartment, that is

$$
\frac{d I}{d t}=\varepsilon E(t)
$$

This group is reduced in two ways. People can either recover or die with the virus or disease. Both options remove people from this group.

$$
\frac{d I}{d t}=\varepsilon E(t)-\mu I(t)-r I(t)-d I(t) .
$$

The population of the recovery group is increased by those that are recovering from the infection. That is,

$$
\frac{d R}{d t}=r I(t)-\mu R(t)
$$

The equation of the death compartment is obtained by removing the expired individuals from the infectious group. This is shown in the equation below;

$$
\frac{d D}{d t}=d I(t)
$$

Hence, the model

$$
\begin{aligned}
& \frac{d S}{d t}=N-\beta S I-\mu S \\
& \frac{d E}{d t}=\beta S I-(\mu+\varepsilon) E \\
& \frac{d I}{d t}=\varepsilon E-(\mu+r+d) I \\
& \frac{d R}{d t}=r I-\mu R \\
& \frac{d D}{d t}=d I \\
& T(t)=S(t)+E(t)+I(t)+R(t),
\end{aligned}
$$

where $T$ is the total population at time $t, N$ is the rate of recruitment, $\beta$ is the rate of infection, $\mu$ is the natural rate of death, $\varepsilon$ is the rate at which a person who is infected becomes prone to infections for each unit of time, $r$ is the rate of recovery of an infected individual per unit time and $d$ is the disease-related death. In this manuscript, we assume a function $\Gamma$, as a policy adopted by the government to alleviate the spread of the epidemic. The policy function is defined by the piecewise function below;

$$
\Gamma(t)=\left\{\begin{array}{ll}
\gamma I, & \text { if } 0 \leq I \leq I_{p} \\
\delta, & \text { if } I>I_{p}
\end{array} .\right.
$$

The death compartment, $D$ do not appear in equation (11), so system (8) can be reduced to the system below 


$$
\begin{aligned}
& \frac{d S}{d t}=N-\beta S I-\mu S \\
& \frac{d E}{d t}=\beta S I-(\mu+\varepsilon) E \\
& \frac{d I}{d t}=\varepsilon E-(\mu+r+d) I-\Gamma(t) \\
& \frac{d R}{d t}=r I-\mu R
\end{aligned}
$$

From system (11),

$$
\begin{gathered}
(T)^{\prime}=N-\mu(T)-(r+d) I-\Gamma \leq \mu(T) \\
\mu(T) \leq N \\
T \leq \frac{N}{\mu} .
\end{gathered}
$$

Then the $\lim _{n \rightarrow \infty} \sup (T) \leq \frac{N}{\mu}$. Therefore, the reasonable region of the reduced system (11) is

$$
\Psi=\left\{(S, E, I, R) \mid \in \mathfrak{R}_{+}^{4}: T \leq \frac{N}{\mu}, S>0, E \geq 0, I \geq 0, R \geq 0\right\} .
$$

The reduced system (11) is considered to be mathematically and epidemiologically reasonable in $\Psi$. We use the concept of the next generation matrix to determine the basic reproductive number [1]. The reduced system (11) always maintains a disease-free equilibrium $x_{0}=\left(\frac{N}{\mu}, 0,0,0\right)$. Near this disease-free equilibrium, $I$ has to be less than $I_{p}$ so the reduced system becomes

$$
\begin{aligned}
& \frac{d S}{d t}=N-\beta S I-\mu S \\
& \frac{d E}{d t}=\beta S I-(\mu+\varepsilon) E \\
& \frac{d I}{d t}=\varepsilon E-(\mu+r+d) I \\
& \frac{d D}{d t}=r I-\mu R
\end{aligned}
$$

\subsection{The Basic Reproduction Number}

An outbreak can be seen as the predicted amount of cases explicitly associated with a particular situation in a population where all the other individuals are prone to infection [13]. In epidemiology, the basic reproduction number, or basic reproductive number is denoted by denoted $R_{0}$ [14]. $R_{0}$ is not a biological constant for the pathogen, because other variables including environmental conditions and the nature of the infected population are also disturbed. Moreover, some driving factors that influence $R_{0}$ are:

the infectivity duration of the patients concerned; the virus' infectiousness and; the number of vulnerable persons in the population with whom the affected patients are connected.

It is also important to know that $R_{0}$ values are usually estimated from mathematical models (in this case the SEIRD model). The basic reproduction number $R_{0}$ does not by itself give an estimate of how fast an infection spreads in the population. It is evident in many infection models [12, 15], $R_{0}>1$ whenever the infection spreads throughout the population, but not if $R_{0}<1$.

Mathematically, the basic reproduction number $R_{0}$ Can be calculated using the following equation: Let $X=(E, I, S)^{T}$. System (16) can be seen as

$$
X^{\prime}(t)=F(X)-V(X),
$$

where

$$
F(X)=\left(\begin{array}{c}
\beta S I \\
0 \\
0
\end{array}\right), \quad V(X)=\left(\begin{array}{c}
(\mu+\varepsilon) E \\
-\varepsilon E+(\mu+r+d) I \\
-N+\beta S I+\mu S
\end{array}\right) .
$$

The Jacobian matrix of both $F(X)$ and $V(X)$ at $X_{0}$ (disease-free equilibrium) are respectively,

$$
D F\left(X_{0}\right)=\left(\begin{array}{ll}
F & 0 \\
0 & 0
\end{array}\right), D V\left(X_{0}\right)=\left(\begin{array}{cc}
V & 0 \\
J_{1} & J_{2}
\end{array}\right),
$$

where $F$ and $V$ are $2 \times 2$ matrices defined as follows

$$
F=\left[\frac{\partial F_{i}\left(X_{0}\right)}{\partial X_{j}}\right] \text { and } V=\left[\frac{\partial V_{i}\left(X_{0}\right)}{\partial X_{j}}\right]
$$

where $i=1,2 ; j=1,2$.

From the above, $F=\left(\begin{array}{cc}0 & \frac{\beta N}{\mu} \\ 0 & 0\end{array}\right)$ and $V=\left(\begin{array}{cc}C_{1} & 0 \\ -\varepsilon & C_{2}\end{array}\right)$,

where $C_{1}=\mu+\varepsilon$ and $C_{2}=\mu+r+d$.

$V$ is a non-singular M matrix. Therefore, it is invertible such that $F V^{-1}=\left(\begin{array}{cc}\frac{\varepsilon \beta N}{\mu C_{1} C_{2}} & \frac{\beta N}{\mu C_{2}} \\ 0 & 0\end{array}\right)$ is the next generation matrix of system (11). The spectral radius of $F V^{-1}$ is given by

$$
\rho\left(F V^{-1}\right)=\frac{\varepsilon \beta N}{\mu C_{1} C_{2}}
$$

Hence, the basic reproduction number of system (11) is

$$
R_{0}=\frac{\varepsilon \beta N}{\mu C_{1} C_{2}}=\frac{\varepsilon \beta N}{\mu(\mu+\varepsilon)(\mu+r+d)} .
$$

\section{Equilibria}

This section discusses the equilibria of system (16). At the 
disease-free equilibria, $X_{0}=\left(\frac{N}{\mu}, 0,0,0\right)$ often occur only if $I \leq I_{p}$. Also, to determine the endemic equilibria of (11), we set the system of equations to 0 as shown

$$
\begin{aligned}
N-\beta S I-\mu S & =0 \\
\beta S I-(\mu+\varepsilon) E & =0 \\
\varepsilon E-(\mu+r+d)-\Gamma(t) & =0 \\
r I-\mu R & =0
\end{aligned}
$$

The above system (23) reduces to

$$
\begin{array}{r}
N-\beta S I-\mu S=0 \\
\beta S I-(\mu+\varepsilon) E=0 \\
\varepsilon E-(\mu+r+d)=0 \\
r I-\mu R=0
\end{array}
$$

if $0 \leq I \leq I_{p}$. When $I>I_{p}$, then system (23) turn out to be

$$
\begin{array}{r}
N-\beta S I-\mu S=0 \\
\beta S I-(\mu+\varepsilon) E=0 \\
\varepsilon E-(\mu+r+d)-\delta=0 \\
r I-\mu R=0
\end{array}
$$

System (24) has a disease-free equilibrium (i.e. $I=0$ ). We solve (24) to determine the endemic equilibria. From the third equation of system (24),

$$
E=\frac{(\mu+r+d)}{\varepsilon} I \text {. }
$$

Substituting the result in the second equation of (24) and solving for $S$, we have

$$
\begin{aligned}
\beta S I & =(\mu+\varepsilon) \frac{(\mu+r+d)}{\varepsilon} I \\
S & =\frac{(\mu+\varepsilon)(\mu+r+d)}{\beta \varepsilon} .
\end{aligned}
$$

Substitution into the first equation of the system in question yields

$$
\begin{aligned}
& I=\frac{\varepsilon N}{(\mu+\varepsilon)(\mu+r+d)}-\frac{\mu}{\beta} \\
& I=\frac{\mu}{\beta}\left(R_{0}-1\right) .
\end{aligned}
$$

Now, solving for $R$,

$$
R=\frac{r}{\beta}\left(R_{0}-1\right)
$$

Back substituting, E becomes

$$
E=\frac{(\mu+r+d)}{\varepsilon} \frac{\mu}{\beta}\left(R_{0}-1\right)
$$

Proposition 1. The system has a distinct disease-free $x_{0}$. If $R_{0}>1$, the system also has a unique endemic equilibrium $X^{*}=\left(S^{*}, E^{*}, I^{*}, R^{*}\right)$, where

$$
\begin{aligned}
S^{*} & =\frac{(\mu+\varepsilon)(\mu+r+d)}{\beta \varepsilon} \\
E^{*} & =\frac{\mu(\mu+r+d)}{\beta \varepsilon}\left(R_{0}-1\right) \\
I^{*} & =\frac{\mu}{\beta}\left(R_{0}-1\right) \\
R^{*} & =\frac{r}{\beta}\left(R_{0}-1\right) .
\end{aligned}
$$

\section{Stability of the Equilibria}

This particular section investigates the stability of the equilibria.

\subsection{Local Stability}

We shall first consider local stability. To do so, we find the Jacobian of system (23). The Jacobian is shown:

$$
J=\left(\begin{array}{cccc}
-\beta I-\mu & 0 & -\beta S & 0 \\
\beta I & -(\mu+\varepsilon) & \beta S & 0 \\
0 & \varepsilon & -(\mu+r+d) & 0 \\
0 & 0 & r & -\mu
\end{array}\right) .
$$

The disease-free equilibrium's local stability is obtained by evaluating the Jacobian at $x_{0}$ :

$$
J=\left(\begin{array}{cccc}
-\mu & 0 & -\beta S & 0 \\
0 & -(\mu+\varepsilon) & \beta S & 0 \\
0 & \varepsilon & -(\mu+r+d) & 0 \\
0 & 0 & r & -\mu
\end{array}\right) .
$$

By subtracting $\lambda$ from the main diagonal, we solve the characteristic equation $\left|J\left(X_{0}\right)-\lambda I\right|=0$. The characteristic equation has two equal roots of $-\mu$. The equation below is solved to find the remaining two roots:

$$
\left|\begin{array}{cc}
-(\mu+\varepsilon+\lambda) & \beta S \\
\varepsilon & -(\mu+r+d+\lambda)
\end{array}\right|=0
$$

Finding the determinant leads to

$$
(\mu+\varepsilon+\lambda)(\mu+r+d+\lambda)-\beta \varepsilon \frac{N}{\mu}=0 .
$$

It is so obvious to see that the equation contains one real root if $R_{0}>1$, and two complex conjugate roots with real part 
when $R_{0}<1$ [15]. This result leads to a proposition.

Proposition 2. If $R_{0}<1$, the disease-free equilibrium $X_{0}<1$ is then locally asymptotically stable.

The stability of the endemic equilibrium $x^{*}$, is gotten from evaluation of the Jacobian at endemic equilibrium [15]. The characteristic equation is shown:

$$
\left|\begin{array}{cccc}
-\beta I-\mu-\lambda & 0 & -\beta S & 0 \\
\beta I & -(\mu+\varepsilon+\lambda) & \beta S & 0 \\
0 & \varepsilon & -(\mu+r+d+\lambda) & 0 \\
0 & 0 & r & -(\mu+\lambda)
\end{array}\right|=0 .
$$

The above characteristic equation has $-\mu$ as a root. The rest of the roots can be found by the characteristic equation below

$$
\left|\begin{array}{ccc}
-\beta I-\mu-\lambda & 0 & -\beta S \\
\beta I & -(\mu+\mathcal{E}+\lambda) & \beta S \\
0 & \varepsilon & -(\mu+r+d+\lambda)
\end{array}\right|=0 .
$$

After the expansion of the determinant and simplifying, we have

$$
\left[\beta I^{*}+\mu+\lambda\right][\mu+\varepsilon+\lambda][\mu+r+d+\lambda]=\beta S^{*} \varepsilon(\mu+\lambda) .
$$

We conclude by Routh-Herwitz criteria [12], that, the eigenvalues of $J\left(X^{*}\right)$ are all negative when $R_{0}>1$.

\subsection{Global Stability $R_{0}>1$}

Theorem 1: If $R_{0}>1$, the endemic equilibrium is then asymptotically stable globally in $\Psi$.

Proof: Consider (8) and $R_{0}>1$, such that the associated unique endemic equilibrium $x^{*}$ of the SEIRD model exist. The global stability of $x^{*}$ is investigated via the Lyapunov function:

$$
V=\left(S-S^{*}-S^{*} \ln \frac{S}{S^{*}}\right)+\left(E-E^{*}-E^{*} \ln \frac{E}{E^{*}}\right)+\left(I-I^{*}-I^{*} \ln \frac{I}{I^{*}}\right) .
$$

We take the derivatives of the function $V$ as shown

$$
\frac{d V}{d t}=\left(1-\frac{S^{*}}{S}\right) \frac{d S}{d t}+\left(1-\frac{E^{*}}{E}\right) \frac{d E}{d t}+\left(1-\frac{I^{*}}{I}\right) \frac{d I}{d t}
$$

Substituting the derivatives $\left(\frac{d S}{d t}, \frac{d E}{d t}, \frac{d I}{d t}\right)$ from (16) into $(40)$

$$
\begin{aligned}
& \frac{d V}{d t}=\left(N-\beta S I-\mu S-\frac{S^{*} N}{S}+S^{*} \beta I+S^{*} \mu\right)+ \\
& \left(\beta S I-C_{1} E-\frac{E^{*} \beta S I}{E}+E^{*} C_{1}\right)+\left(Q \varepsilon E-Q C_{1} I-\frac{Q I^{*} \varepsilon E}{I}+Q I^{*} C_{2}\right) .
\end{aligned}
$$

At the steady-state,

$$
N-\beta S^{*} I^{*}+\mu S^{*} \text {. }
$$

Substituting (42) into (41) yields:

$$
\begin{aligned}
& \frac{d V}{d t}=\left(\beta S^{*} I^{*}+\mu S^{*}-\beta S I-\mu S-\frac{S^{*} \beta S^{*} I^{*}}{S}-\frac{S^{*} \mu S^{*}}{S}+S^{*} \beta I+S^{*} \mu\right)+ \\
& \left(\beta S I-C_{1} E-\frac{E^{*} \beta S I}{E}+E^{*} C_{1}\right)+\left(Q \varepsilon E-Q C_{1} I-\frac{Q I^{*} \varepsilon E}{I}+Q I^{*} C_{2}\right) .
\end{aligned}
$$

After simplifying we get:

$$
\begin{aligned}
& \frac{d V}{d t}=\left(\beta S^{*} I^{*}+\mu S^{*}-\mu S-\frac{S^{* 2} \beta I^{*}}{S}-\frac{S^{* 2} \mu}{S}+S^{*} \beta I+S^{*} \mu\right)+ \\
& \left(-C_{1} E-\frac{E^{*} \beta S I}{E}+E^{*} C_{1}\right)+\left(Q \varepsilon E-Q C_{1} I-\frac{Q I^{*} \varepsilon E}{I}+Q I^{*} C_{2}\right) .
\end{aligned}
$$

Grouping the terms with infected class without the $(*)$ from (44) and equating to 0 , we obtain

$$
S^{*} \beta I-C_{1} E+Q \varepsilon E-Q C_{2} I=0 \text {. }
$$

A little perturbation of the steady-state from (16) and (45) yields:

$$
Q=\frac{S^{*} \beta}{C_{2}}, C_{1}=\frac{\beta S^{*} I^{*}}{E^{*}}, \varepsilon=\frac{C_{2} I^{*}}{E^{*}} .
$$

Substituting (46) into (44) and performing algebraic calculation results in:

$$
\begin{aligned}
& \frac{d V}{d t}=\beta S^{*} I^{*}+\mu S^{*}-\mu S-\frac{S^{* 2} \beta I^{*}}{S}-\frac{S^{* 2} \mu}{S}+S^{*} \mu- \\
& \frac{E^{*} \beta S I}{E}+S^{*} I^{*} \beta-\frac{I^{* 2} E S^{*} \beta}{I E}+S^{*} \beta I^{*} .
\end{aligned}
$$

Factorization of (45) gives:

$$
\frac{d V}{d t}=\mu S^{*}\left(2-\frac{S}{S^{*}}-\frac{S^{*}}{S}\right)+\beta S^{*} I^{*}\left(3-\frac{S^{*}}{S}-\frac{I^{*} E}{I E^{*}}-\frac{S E^{*} I}{E}\right) .
$$

Because the arithmetic mean exceeds the geometric mean, the inequality in (48) holds:

$$
\left(2-\frac{S}{S^{*}}-\frac{S^{*}}{S}\right) \leq 0, \quad\left(3-\frac{S^{*}}{S}-\frac{I^{*} E}{I E^{*}}-\frac{S E^{*} I}{E}\right) \leq 0 .
$$

This implies $\frac{d V}{d t} \leq 0$ for $R_{0}>1$. Therefore, $V$ is a Lyapunov function in $\Psi$ and by the LaSalle's invariance principle [12], every solution to equation (16) approaches the associated unique endemic equilibria $x^{*}$, of the SEIRD model as $t \rightarrow \infty$ for which $R_{0}>1$. 


\section{Results and Discussion}

With the SEIRD model, we can predict the outcome of a decision in different situations. Our numerical simulations were done with MATLAB 2018a. The predictions are based on a decision taken by the government to alleviate the spread of the virus. We simulate the model by using the parameters from the recent data of worldometer, 2020 which can be obtained from http://www.worldometers.info/coronavirus [16].

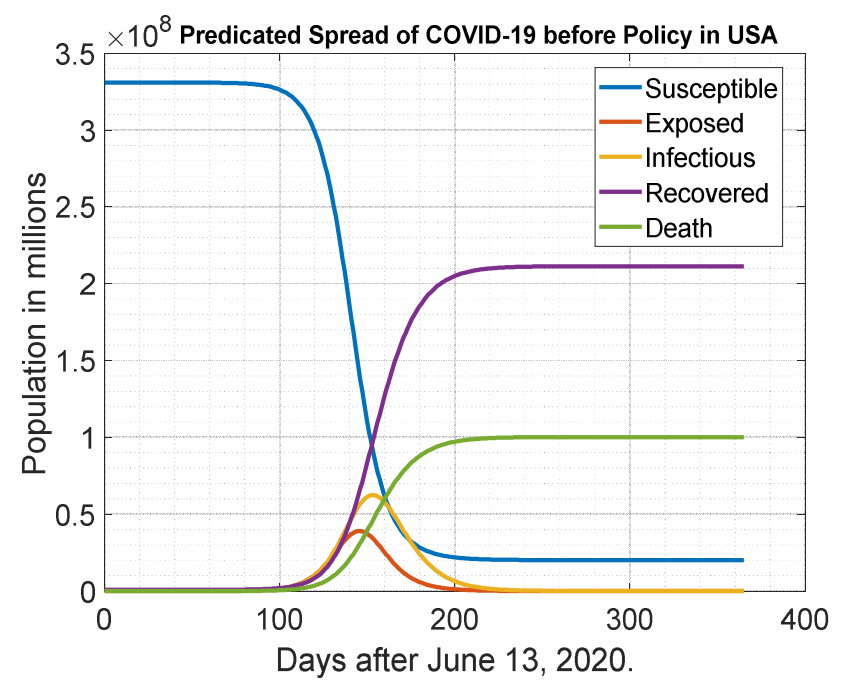

(a) Prediction before policy

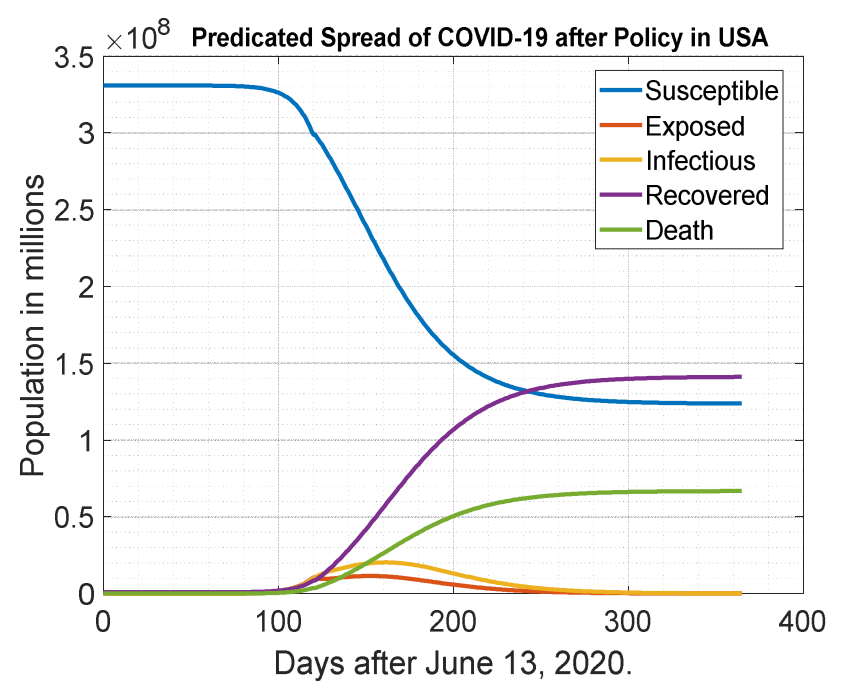

(b) Prediction after policy

Figure 2. A numerical solution to the SEIRD model before and after the interventional policy by the government of the United States of America.

Per the data as at June 13, 2020, the recruitment rate $(N)$ of the United States is $3.30906997 e+08, \quad \beta=9.4980 \mathrm{e}-10, \quad \mu$ is assumed to be $0, \quad \varepsilon=0.1923, \quad d=0.0340, \quad r=0.1429$, $S(0)=330,906,892, \quad E(0)=5.2, \quad I(0)=105, \quad R(0)=841,936 \quad$ and $D(0)=6$. Figure 2 shows the graphical illustration of the SEIRD model for the next 365 days as at June 13, 2020. We compare the predictions of the spread of the pandemic before and after the government's policy.

Table 1. Prediction of the Last Five Days Before Government Policy.

\begin{tabular}{llllll}
\hline Day & Susc. & Exp. & Infec. & Rec. & Death \\
\hline 361 & 20126624 & 7.0 & 43.5 & 211390541 & 100221142 \\
362 & 20126623 & 6.5 & 40.4 & 211390544 & 100221143 \\
363 & 20126622 & 6.1 & 37.54 & 211390547 & 100221145 \\
364 & 20126621 & 5.64 & 34.9 & 211390549 & 100221146 \\
365 & 20126621 & 5.2 & 32.4 & 211390552 & 100221147 \\
\hline
\end{tabular}

Table 2. Prediction of the Last Five Days After Government Policy.

\begin{tabular}{llllll}
\hline Day & Susc. & Exp. & Infec. & Rec. & Death \\
\hline 361 & 123765716 & 31470 & 84738 & 141095656 & 66760777 \\
362 & 123760819 & 30419 & 81903 & 141101607 & 66763610 \\
363 & 123756085 & 29403 & 79161 & 141107360 & 66766348 \\
364 & 123751511 & 28420 & 76512 & 141112920 & 66768994 \\
365 & 123747090 & 27469 & 73953 & 141118293 & 66771552 \\
\hline
\end{tabular}

Data about the outbreak of COVID-19 in the United States was obtained from worldometer. The spread of the outbreak was studied with the SEIRD model from June 13, 2020 to June 12, 2021 (a period of one year).

Table 1 and Table 2 show the last five days (i.e. 361-365) of the predicted data. From Table 1, the number of the susceptible population decreases steadily as the death population increases very rapid without the government's interventional policy (i.e. social distancing, wearing of nose mask, frequently washing of hands with soap under running water, etc.). Table 2 also describes the decline of the number of deaths and a rise in the number of susceptible after the introduction of the interventional policy by the government. This depicts that the spread of the disease will diminish in the next 365 days after the introduction of the policy rendering the model effective.

\section{Conclusion}

In this manuscript, a simple SEIRD epidemic model was proposed and analyzed with an intervention of government policy to simulate and predict the spread of the coronavirus pandemic. This model was theoretically studied to examine the global stability of the equilibria by the use of Lyapunov function. Each time the associated reproduction number is less than one $\left(R_{0}<1\right)$ or greater than one $\left(R_{0}>1\right)$, the disease-free and endemic equilibria respectively were seen to be globally asymptotic.

There is an indication that, for the endemic equilibrium, the disease will linger in the population if it is not controlled in the population. Future research will explore on vaccinated models for treating COVID-19.

\section{Acknowledgments}

The authors acknowledge the following persons for their constant review of the manuscript; Dr. Jerry John Kponyo, Kwame Obour Opuni-Boachie Agyemang, Elijah Asante Boakye, Justice Owusu Agyemang and Fred Appiah-Twum. 


\section{References}

[1] P. Van den Driessche, and J. Watmough, "Reproduction numbers and sub-threshold endemic equilibria for compartmental models of disease transmission," Mathematical biosciences, vol 180 (1-2), pp. 29-48, 2002.

[2] M. M. Ojo, and F. O. Akinpelu, "Lyapunov Functions and Global Properties of SEIR Epidemic Model," International journal of Chemistry, Mathematics and Physics, vol 1 (1), 2017.

[3] C. Wang, P. W. Horby, F. G. Hayden, and G. F. Gao, "A novel coronavirus outbreak of global health concern", The Lancet, vol 395 (10223), pp. 470-473, 2020.

[4] R. M. May, and R. M. Anderson, "Population biology of infectious diseases: Part II”, Nature, vol 280 (5722), pp. 455-461, 1979.

[5] B. Ivorra, B. Martínez-López, J. M. Sánchez-Vizcaíno, and Á. M. Ramos, "Mathematical formulation and validation of the Be-FAST model for Classical Swine Fever Virus spread between and within farms", Annals of operations research, vol 219 (1), pp. 25-47, 2014.

[6] B. Martínez-López, B. Ivorra, Á. M. Ramos, and J. M. Sánchez-Vizcaíno, "A novel spatial and stochastic model to evaluate the within-and between-farm transmission of classical swine fever virus. I. General concepts and description of the model”, Veterinary microbiology, vol 147 (3-4), pp 300-309, 2011.

[7] H. R. Thieme, Mathematics in population biology, vol. 12, Princeton University Press, 2018.

[8] F. Brauer, and C. Castillo-Chavez, Mathematical models in population biology and epidemiology, vol. 2, New York: Springer, 2012.

[9] D. Yan, and H. Cao, "The global dynamics for an age-structured tuberculosis transmission model with the exponential progression rate", Applied Mathematical Modelling, vol 75, pp. 769-786, 2019.

[10] K. Roosa, Y. Lee, R. Luo, A. Kirpich, R. Rothenberg, J. M. Hyman,... and G. Chowell, "Real-time forecasts of the COVID-19 epidemic in China from February 5th to February 24th, 2020", Infectious Disease Modelling, vol 5, pp. 256-263, 2020.

[11] A. J. Kucharski, T. W. Russell, C Diamond, Y. Liu, J. Edmunds, S. Funk,... and N. Davies, "Early dynamics of transmission and control of COVID-19: a mathematical modelling study", The lancet infectious diseases, 2020.

[12] S. A. Al-Sheikh, "Modeling and analysis of an SEIR epidemic model with a limited resource for treatment", Global Journal of Science Frontier Research Mathematics and Decision Sciences, vol 12 (14), pp. 56-66, 2012.

[13] C. Fraser, C. A. Donnelly, S. Cauchemez, W. P. Hanage, M. D. Van Kerkhove, T. D. Hollingsworth,... and T. Jombart, "Pandemic potential of a strain of influenza A (H1N1)", Early findings science, vol 324 (5934), 1557-1561, 2009.

[14] G. N. Milligan, and A. D. Barrett, Vaccinology: An Essential Guide, Wiley Blackwell, February, 2015.

[15] M. Martcheva, An introduction to mathematical epidemiology,

vol. 61. New York: Springer, 2015.

[16] Worldometers, COVID-19 Coronavirus Pandemic. Dover, Delaware, U.S.A, June, 2020. http://www.worldometers.info/c-oronavirus.

\section{Biography}

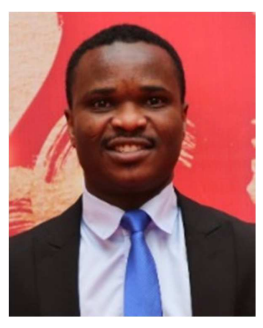

Joseph Roger Arhin obtained his BSc degree in Mathematics from the University of Education, Winneba, Ghana, in 2017 and received the MSc degree in Mathematics (Numerical Linear Algebra and Scientific Computing with Application) from University of Electronic Science and Technology of China, Chengdu, China, in 2020. He will be starting his $\mathrm{PhD}$ course in Information and Communication Engineering at University of Electronic Science and Technology of China, Chengdu, China, in September 2020. His PhD research will focus on machine learning and artificial intelligence.

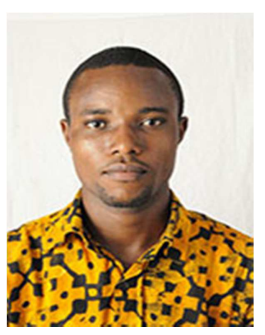

Francis Sam received the BSc degree in Mathematics from the University of Education Winneba, Ghana, in 2017. He is currently pursuing the MSc. degree in Mathematics with the School of Mathematical Sciences from the University of Electronic Science and Technology of China. He has a long-term interest in tensor decompositions and their practical applications in massive MIMO. His broad research interests include Tensors and wireless communication.

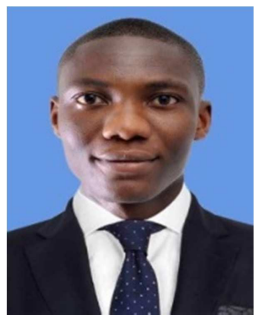

Kenneth Coker had his BSc degree in Telecommunication Engineering from the Kwame Nkrumah University of Science and Technology, Ghana, in 2017 and obtained his MSc degree in Information and Communication Engineering from University of Electronic Science and Technology of China, Chengdu, China, in 2020. He is planning to commence his $\mathrm{PhD}$ course in Information and Communication Engineering at University of Electronic Science and Technology of China, Chengdu, China, in September 2020.

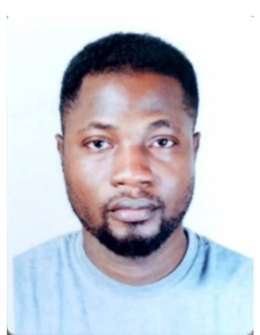

China, 2020.
Ernest Owusu Ansah attained his Bachelor's degree in Economics and Statistics from the Garden City University College, Ghana in 2015. He had his MSc degree in Mathematics with a research area of Numerical Solution to Differential and Integral equations from University of electronic science and technology of 